\title{
Éditorial:
}

\section{L'enseignement de la gérontologie - L'éveil d'un géant endormi}

L'année 1989 a vu la naissance d'un groupe d'intérêt sur l'enseignement de la gérontologie au sein de l'Association canadienne de gérontologie (ACG). Dès 1990, plus de 140 membres de l'ACG s'identifiaient au groupe et 17 articles avaient été présentés (Thornton, 1992). Le groupe s'est développé à un point tel qu'en 1996, le conseil de l'ACG approuvait la formation d'une division de gérontologie éducative.

En 1991, le Conseil consultatif national sur le 3e âge (CCNTA) a rendu publique sa position sur la formation en gérontologie composée de 15 recommandations et prônant les services au système actuel. Les recommandations du CCNTA mettaient entre autres l'accent sur les besoins en personnel et le développement de programmes, et plus particulièrement sur les prévisions en ressources humaines, un relevé national de la formation en gérontologie, les directives en matière de développement de programmes, la formation des professeurs et les stratégies de promotion de l'intérêt des étudiants. On signale certains progrès. L'ACG a préparé un relevé national des programmes en gérontologie (Association canadienne de gérontologie, 1994). Les activités entreprises conjointement avec le Centre d'éducation sur le vieillissement et la santé ont donné lieu à l'établissement de lignes directrices pour les programmes en art dentaire, en travail social, en sciences infirmières, en réadaptation et en médecine (Ontario University Coalition for Education in Health Care of the Elderly, 1993).

Le document du CCNTA encourage également la formation permanente de toutes les personnes dont les tâches sont étroitement reliées à la vie des aînés. Il souligne le besoin d'ingéniosité de la part des prestateurs de services qui doivent souvent faire face à des circonstances hors du commun dans le cadre de leur travail. On est peut-être en train de dire que les ateliers d'un jour tenus hors des lieux de travail se marient plus ou moins bien aux modes de financement actuels.

Le CCNTA recommandait que l'éducation en gérontologie adopte une perspective multidisciplinaire, opinion largement répandue, en théorie du moins. Il recommandait aussi qu'au Canada, elle tienne compte de la diversité culturelle des aînés et de leur famille, une dimension connue aux États-Unis sous le nom d'ethnogériatrie. Il suggérait aux employeurs de reconnaître la formation en gérontologie et au gouvernement ainsi qu'au secteur privé d'appuyer le développement de l'éducation dans le domaine du vieillissement. Enfin, il recommandait la mise sur pied de programmes visant à aider les intervenants des services formels à répondre aux besoins 
des personnes de soutien informel et soulignait l'importance de la défense des droits des aînés.

En 1992, Jim Thornton a proposé une taxonomie de l'éducation sur le 3e âge au Canada. S'appuyant sur le travail de Peterson (1980), il a maintenu que la gérontologie au Canada se divisait en trois secteurs : la formation des aînés, c'est-à-dire le secteur qui se penche sur la formation des aînés; la formation en gérontologie, soit la formation des jeunes et du grand public et enfin, la formation universitaire en gérontologie, soit la formation et l'enseignement menant à un diplôme ou à une spécialisation. Il voulait par là diviser la science gérontologique en fonction de l'auditoire ou de l'apprenant avant tout. Tout comme Peterson, Thornton séparait l'étude et la recherche de la pratique. Bien que notre éditorial traite d'abord de la formation universitaire, surtout en ce qui concerne les professionnels de la santé, il est important de reconnaître l'ensemble du secteur et d'intégrer ses trois composantes à l'ACG. Incidemment, Thornton avait fait une mise en garde sur la déviation éventuelle de la gérontologie vers les enjeux de santé. Son apport a contribué à la clarification de la pensée en matière d'élargissement de la formation en gérontologie et a fait ressortir l'importance d'une approche d'éducation permanente.

En 1995, le Bureau of Health Professions of the Health Resources and Services Administration (États-Unis) a publié A National Agenda for Geriatric Education: White Papers (Klein, 1995) suivi, un an plus tard, du compte rendu d'un forum national traitant des White Papers (Klein, 1996). Les cadres supérieurs du service américain de santé publique considéraient la formation en gériatrie comme un "géant endormi". Les White Papers ont considérablement fait avancer la cause de la gériatrie tant sur le plan pratique que conceptuel. Sur le plan pratique, le document détermine les dispositions à prendre, les agents responsables, les résultats qu'on peut espérer et même les délais souhaitables. Sur le plan conceptuel, on y trouve une approche systémique beaucoup plus précise que celle du CCNTA et une proposition de thèmes génériques divisée en disciplines spécifiques. La gestion des soins, les soins de longue durée, la prise en charge de cas, la formation interdisciplinaire et l'ethnogériatrie constituent les thèmes génériques. Les White Papers présentent une division des thèmes génériques en cinq disciplines spécifiques, soit l'art dentaire, la médecine, les soins infirmiers, la santé publique et le travail social. Un chapitre moins dense traite des quelque 200 professions connexes au domaine de la santé qui constituent 60 pour cent de la main d'ouvre des soins de santé.

Étant donné que la formation universitaire en gérontologie comprend à la fois l'étude (enseignement et recherche) et l'intervention, il faudra orienter les efforts vers ces deux secteurs. Sur le plan de l'étude, il faudra attirer les étudiants d'abord en leur faisant miroiter le revenu potentiel, en rendant les emplois attrayants et en imposant ou en récompensant les compétences supplémentaires, conformément aux recommandations du 
CCNTA. Il faut rendre l'expérience de l'apprentissage aussi stimulante que possible en formant et en recrutant des professeurs compétents. Ce sont là certains des principes de base de la position du CCNTA.

La gérontologie éducative offre des retombées avantageuses au monde de l'enseignement supérieur qui vont bien au-delà du domaine du vieillissement. Toute maison canadienne d'enseignement postsecondaire devra inclure en priorité à son programme d'enseignement professionnel, le travail d'équipe multidisciplinaire enseigné dans un cadre multidisciplinaire et se concentrer sur les diversités culturelles.

Ni l'exposé des principes du CCNTA ni les White Papers américains ne traitent de l'effet des valeurs sur le comportement professionnel, le travail d'équipe et les méthodes des établissements. Nous devons examiner la façon dont les valeurs influencent les relations des professionnels entre eux et envers leurs clients. Clark (1996) a signalé que les valeurs varient d'une profession à l'autre. Il prétend que la médecine, par exemple, embrasse des valeurs scientifiques et humanistes. Les membres de la profession infirmière prônent la dignité humaine tandis que les travailleurs sociaux favorisent la revendication et la prise en charge de soi. Bien sûr, un tel énoncé constitue une simplification à outrance qui ne rend pas justice au travail de Clark mais il a le mérite d'illustrer le défi de la formation interdisciplinaire puisque les professions ne respectent pas toujours les valeurs des autres professions. D'autre part, la mise en commun des valeurs au sein d'une équipe peut produire des résultats beaucoup plus puissants que ne le ferait une profession isolée. L'apprentissage du travail d'équipe demeure un défi de taille en gérontologie.

Au-delà des valeurs individuelles et professionnelles, l'étude de la gérontologie doit adopter une nouvelle vision des soins de longue durée. D'abord, nous devons adopter une définition de la santé en termes de promotion de la santé et l'appliquer aux aînés. En résumé, la définition stipule que la santé est une ressource de vie qui permet aux individus de réaliser leurs aspirations et de composer avec leur environnement ou de le changer au besoin. Or, trop souvent, nous aidons à faire face aux situations mais nous laissons tomber l'aide à la réalisation des aspirations ou au changement de l'environnement.

En deuxième lieu, comme l'ont proposé Lidz, Fisher et Arnod (1992), nous devons, dans le cadre des soins de longue durée, séparer la fonction médicale de la fonction résidentielle dans les centres d'accueil ou ailleurs. Il faut se départir de l'idée que les aînés qui ont quelque forme d'invalidité sont des patients à temps plein qui doivent rigoureusement suivre les ordres des soignants. Les auteurs suggèrent que le modèle hospitalier soit remplacé par un modèle d'hospitalité.

Troisièmement, nous devons établir une culture ou un climat au sein des établissements de services qui ne sont pas engagés dans des activités de formation pour les amener à appuyer les processus d'apprentissage. French (1995) a prétendu que la culture universitaire, qui favorise la 
remise en question de l'autorité, le changement, l'innovation et l'élaboration de théories pourrait bien se heurter à la culture des services qui valorise la pratique et l'expérience au détriment de la théorie et de l'innovation. Il nous faut une troisième culture qui saura relier les deux premières ou du moins fera naître la tolérance à leur égard.

Une fois engagée dans cet important programme de réforme, la gérontologie éducative devra examiner sa propre viabilité, ce qui exigera une analyse détaillée du cadre législatif et politique, de la structure institutionnelle, des ressources techniques, financières et d'infrastructure, ainsi que des ressources humaines (Shahi, Hartvelt \& Sacks, 1997). Ce sont les éléments pertinents pour le développement de programmes éducatifs viables en matière de vieillissement. Les White Papers constituent un travail remarquable à cet égard.

La gérontologie éducative s'éveille et s'apprête à prendre son envol. Ses trois secteurs prendront de la force grâce au travail d'équipe. L'éducation des aînés mêmes, l'éducation du grand public au sujet des aînés et la formation des professionnels permettant une meilleure compréhension de l'aîné malade ou en santé représentent trois dimensions dont la réunion constituera le fondement de programmes éducatifs en matière de vieillissement répondant aux besoins actuels de notre société.

\section{Références}

Association canadienne de gérontologie. (1994). Programmes et cours en gérontologie et gériatrie en établissements post-secondaires au Canada. Ottawa, ON: Association canadienne de gérontologie.

Clark, P.G. (1996). Diversity in heath care professional socialization: Implications for geriatric education in interdisciplinary teamwork. Paper presented at the 22nd annual meeting of the Association for Gerontology in Higher Education, Philadephia, PA.

Conseil consultatif national sur le troisième âge. (1991). La position du CCNTA sur la formation en gérontologie. Ottawa, ON: Approvisionnements et Services Canada (Cat. No. H71-212-11-1991).

French, S.E. (1995). Creating a CTU Culture. News from the CTU's. Hamilton, ON: Interdisciplinary Aging and Health Program, McMaster University.

Klein, S.N. (Éd.). (1995). A national agenda for geriatric education: White papers. Rockville, MD: Bureau of Health Professions, Interdisciplinary Geriatrics and Allied Heath Branch.

Klein, S.M. (Éd.). (1996). A national agenda for geriatric education: Forum report. Rockville, MD: Bureau of Heath Professions, Interdisciplinary, Geriatrics and Allied Health Branch.

Lidz, C.W., Fisher, L.I., \& Arnod, R.M. (1992). The erosion of autonomy in long term care. New York: Oxford University Press.

Ontario University Coalition for Education in Health Care of the Elderly. (1993). Educational development and curriculum content in aging and health: Guidelines for health professionals in Ontario. Hamilton, ON: McMaster University, Educational Centre for Aging and Health. 
Peterson, D.A. (1980). Who are the educational gerontologists? Educational Gerontology, 5, 311-323.

Shahi, G.S., Hartvelt, F., \& Sacks, M. (1997). Realizing sustainable public health: Implications for public health training. In G.S. Shahi, B.S. Levy, A. Binger, T. Kjellstrom, \& R. Lawrence (Eds.), International perspectives on environment, development and health: towards a sustainable world (pp. 697-716). New York: Springer Publishing Company.

Thornton, J.E. (1992). Educational gerontology in Canada. Educational Gerontology, 18, 415-441.

Alexander Macpherson 